Studies on sampling and homogeneous dual readout calorimetry with meta-crystals

This article has been downloaded from IOPscience. Please scroll down to see the full text article.

2011 JINST 6 P10012

(http://iopscience.iop.org/1748-0221/6/10/P10012)

View the table of contents for this issue, or go to the journal homepage for more

Download details:

IP Address: 137.138.124.233

The article was downloaded on 26/07/2012 at $16: 19$

Please note that terms and conditions apply. 


\section{Studies on sampling and homogeneous dual readout calorimetry with meta-crystals}

\section{G. Mavromanolakis, ${ }^{1}$ E. Auffray and P. Lecoq}

European Organization for Nuclear Research, CERN, CH-1211 Geneva, Switzerland

E-mail: Georgios. Mavromanolakis@cern.ch

ABSTRACT: The meta-crystals concept is an approach that consists of using both undoped and properly doped heavy crystal fibers of identical material as the active medium of a calorimeter. The undoped fibers behave as Cherenkov radiators while the doped ones behave as scintillators. A dual readout calorimeter can be built with its sensitive volume composed of a mixture of both types of crystals. In addition if the calorimeter is adequately finely segmented it can also function as a particle flow calorimeter at the same time. In this way one could possibly combine the advantages of both the particle flow concept and the dual readout scheme. We discuss the approach of dual readout calorimetry with meta-crystals made of Lutetium Aluminium Garnet (LuAG). We briefly present studies on the material development and first testbeam activities and then focus on performance expectation studies based on simulation. We discuss in more detail the results from generic systematic scannings of the design parameters of a dual readout calorimeter. The parameters under study include the transverse and longitudinal granularity, the sampling frequency and readout fraction of the scintillation and the Cherenkov signals, the total calorimeter length, the mixture of homogeneous and sampling dual readout components, their corresponding composition etc. We close with a brief outlook on open issues and further R\&D needed to proceed from an ideal conceptual case to the design of a realistic detector.

KEYWORDS: Calorimeters; Calorimeter methods; Detector modelling and simulations I (interaction of radiation with matter, interaction of photons with matter, interaction of hadrons with matter, etc)

\footnotetext{
${ }^{1}$ Corresponding author.
} 


\section{Contents}

1 Introduction 1

2 Calorimetry with meta-crystals $\quad 1$

2.1 Material development and related studies 2

2.2 Testbeam activities 4

$\begin{array}{lll}2.3 & \text { Simulation studies } & 6\end{array}$

$\begin{array}{llr}3 & \text { Outlook } & 8\end{array}$

\section{Introduction}

An experiment at a future lepton collider sets strict requirements for vertex, tracking and calorimetric detectors. The R\&D effort on the design of the calorimeters is directed mainly into two concepts, the particle flow approach [1] and the dual readout scheme [2]. Within the former both the electromagnetic and the hadronic calorimeters are very finely segmented in order to allow very efficient pattern recognition and thus identify and track all particles in a jet. In the dual readout approach one would like to have a calorimeter that is capable of measuring both the ionisation or scintillation and the Cherenkov signals generated by a hadronic shower. By doing so it is possible to determine on an event by event basis the electromagnetic fraction of the shower in order to correct for this source of fluctuation that degrades the energy resolution of the calorimeter. In the following we discuss a dual readout approach that is based on meta-crystals [3].

\section{Calorimetry with meta-crystals}

The meta-crystals concept consists of using both undoped and properly doped heavy crystal fibers of identical material for the generation of the signal of the detector. The undoped fibers behave as Cherenkov radiators while the doped ones behave as scintillators. A dual readout calorimeter can be built with its sensitive volume composed of a mixture of both types of crystals. In addition if the readout volume is adequately finely segmented longitudinally and transversely it can also function as a particle flow calorimeter at the same time. The conceptual design of a readout unit of such a calorimeter is illustrated in figure 1 . The unit consists of a structured distribution of different types of fibers. Typical dimensions of a unit are of the order of $1-1.5 R_{M}$ and $20-25 X_{0}$ in the transverse and longitudinal direction respectively. In addition to Cherenkov and scintillating fibers one could also have fibers that would be sensitive to neutrons and so to have a triple readout calorimeter. Candidate materials are those based on lithium or boron which have high neutron capture cross-section. As shown in the figure the light from the different types of fibers is directed to different silicon photomultipliers or other solid state photodetectors by using diffractive optics light concentrators or micro-lenses. The diffractive optics plate is patterned so to match the structure of 


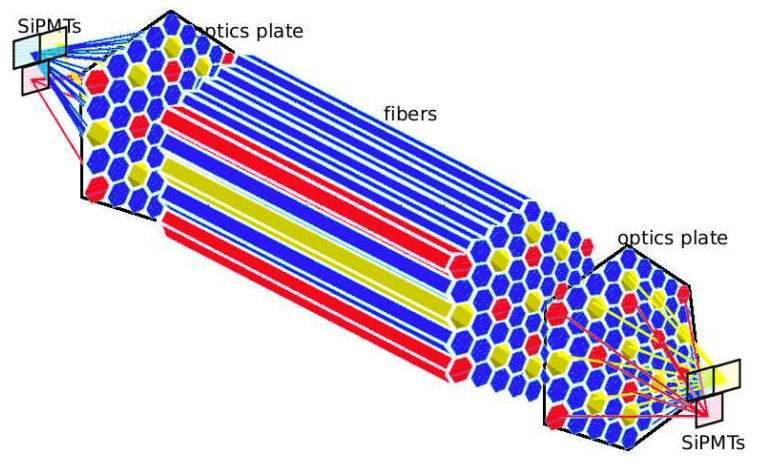

Figure 1. Conceptual design of a readout unit composed of a structured distribution of three different types of crystal fibers, Cherenkov, scintillating and neutron sensitive. The light from the different types of fibers is directed to different silicon photomultipliers by micro-lenses plates at both ends of the unit. (Not to scale)

Table 1. Physical and optical properties of Lutetium Aluminium Garnet (LuAG) crystal.

\begin{tabular}{|llll|}
\hline Physical properties & \multicolumn{3}{c|}{ Optical properties } \\
\hline Density & $6.73 \mathrm{gr} / \mathrm{cm}^{3}$ & Light yield (Ce doped) & $25000 \mathrm{ph} / \mathrm{MeV}$ \\
Zeff & 62.9 & Emission wavelength & $520 \mathrm{~nm}(\mathrm{Ce}$ doped) \\
Radiation length $X_{0}$ & $1.41 \mathrm{~cm}$ & Decay time & $60 \mathrm{nsec}(\mathrm{Ce}$ doped) \\
Interaction length $\lambda_{I}$ & $23.3 \mathrm{~cm}$ & Refractive index & $1.842 \mathrm{at} 633 \mathrm{~nm}$ \\
Melting point & $2260{ }^{\circ} \mathrm{C}$ & Cherenkov threshold & $97 \mathrm{keV}$ \\
Thermal expansion & $8.810^{-6} /{ }^{\circ} \mathrm{C}$ & Max Cherenkov angle & $57^{\circ}$ \\
Thermal conductivity & $31 \mathrm{~W} / \mathrm{m}^{\circ} \mathrm{C}$ & Total reflection angle & $33^{\circ}$ \\
\hline
\end{tabular}

fibers and can be attached to both ends of the unit. The development of such a concept requires a comprehensive program of R\&D studies in various areas, from material development and crystal fiber production to simulation studies, testbeam activities and prototyping. Recent progress on these topics is discussed below.

\subsection{Material development and related studies}

A candidate material under study to be used for this concept is the Lutetium Aluminium Garnet $\mathrm{Lu}_{3} \mathrm{Al}_{5} \mathrm{O}_{12}$ ( $\mathrm{LuAG}$ ) crystal. Its properties are summarised in table 1 . This crystal material combines a high density of $6.73 \mathrm{gr} / \mathrm{cm}^{3}$ and relatively short radiation and interaction lengths of $1.41 \mathrm{~cm}$ and $23.3 \mathrm{~cm}$ respectively sufficient to build a dense and compact detector. Its high refractive index of 1.842 at $633 \mathrm{~nm}$ and low energy threshold for Cherenkov radiation at $97 \mathrm{keV}$ for electrons makes it a good Cherenkov radiator (to be compared with 1.55 and $190 \mathrm{keV}$ respectively for quartz). Furthermore when LuAG is doped with cerium $(\mathrm{Ce})$ it shows excellent scintillating performance with high light yield of about 25000 photons per deposited $\mathrm{MeV}$, emission peaked at $520 \mathrm{~nm}$ and decay time of about 60 nsec. A faster scintillation signal can be achieved if LuAG is doped with praseodymium $(\mathrm{Pr})$, then the scintillation signal is characterized by UV emissions peaked at $320 \mathrm{~nm}$ and $370 \mathrm{~nm}$ and decay time of $20 \mathrm{nsec}$ (see figure 4 and [5]). 


\section{Micro-pulling down method}

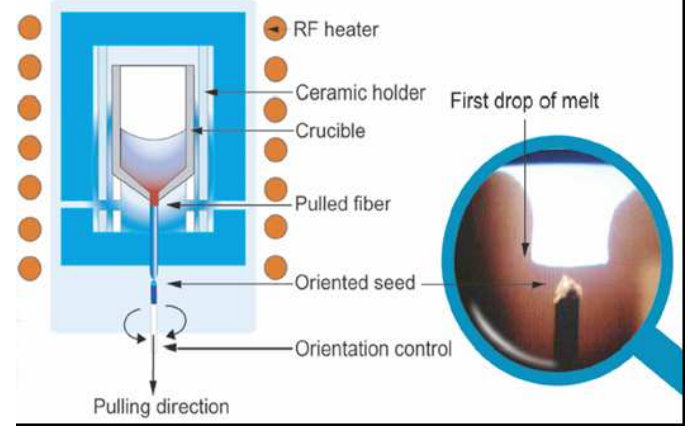

Figure 2. Schematic of the micro-pulling down method used in the production of crystal fibers.

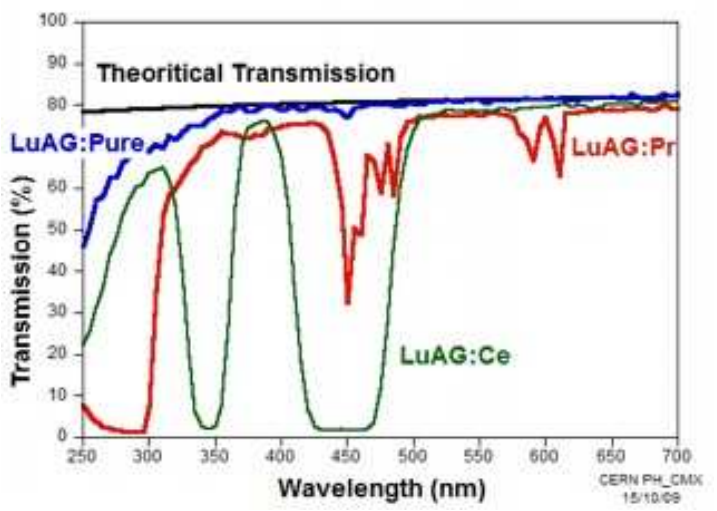

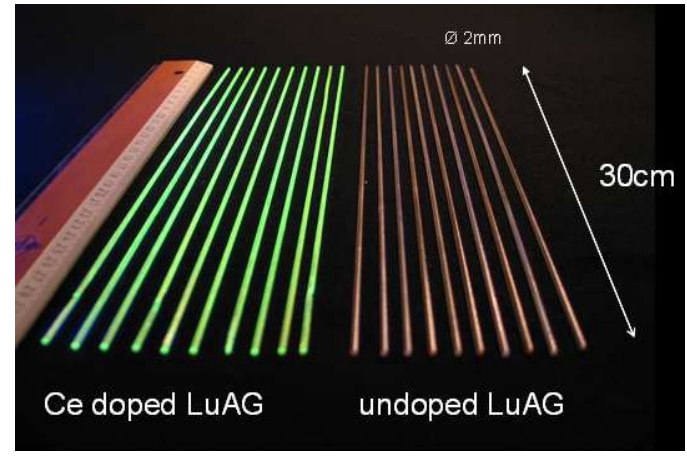

Figure 3. Samples of Ce doped and undoped LuAG crystal fibers produced by FibercrystLyon with the micro-pulling down method.

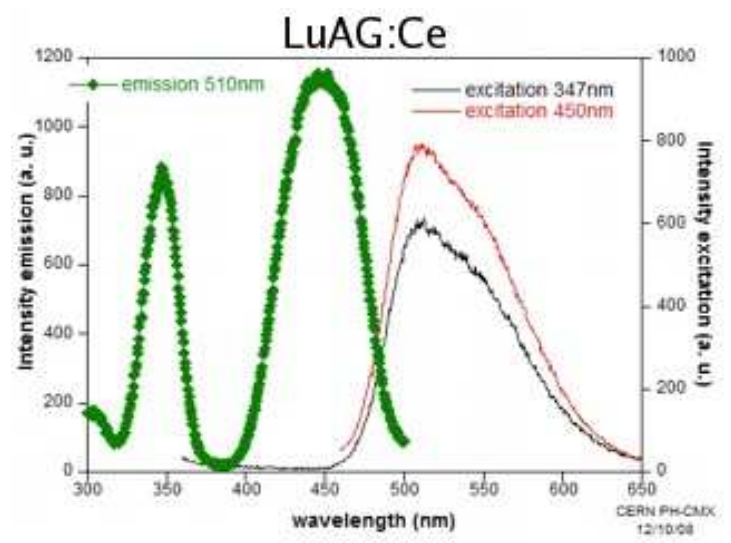

Figure 4. Illustration of series of measurements for optical characterisation of crystal fibers.

Bulk LuAG crystals can be produced with traditional growth methods like the Czochralski or Bridgman-Stockbarger technique. The ingot produced then undergoes cutting and polishing procedures to the desired shape and dimensions. With these methods it is very difficult to produce fibers or in general elongated crystals with large aspect ratio. A technology that can be used for this purpose is the micro-pulling down method, a schematic of which is shown in figure 2. The University of Lyon1 in collaboration with Fibercryst-Lyon have improved the method and applied it successfully to the production of fibers made of LuAG and other crystal materials [4]. With this method crystal fibers with diameter between 0.3 and $3 \mathrm{~mm}$ and length up to $2 \mathrm{~m}$ can be produced. The pulling rate is ranging from 0.1 to $0.5 \mathrm{~mm} / \mathrm{min}$ and is considerably faster than the traditional methods, about a factor 10 and 50 times faster than Czochralski and Bridgman-Stockbarger method respectively. Another advantage of this technique is the fact that the capillary die can be non-cylindrical e.g. square, hexagonal etc, and so fibers with these shapes in cross-section can be produced as well. The method is yet to be optimised further for industrial application and it is expected that its overall cost per unit volume of production will be comparable to that of standard crystal growth methods. Samples of Ce doped and undoped LuAG crystal fibers, $2 \mathrm{~mm}$ in diameter and $30 \mathrm{~cm}$ in length, produced with this method are shown in figure 3. Figure 4 shows a typical 

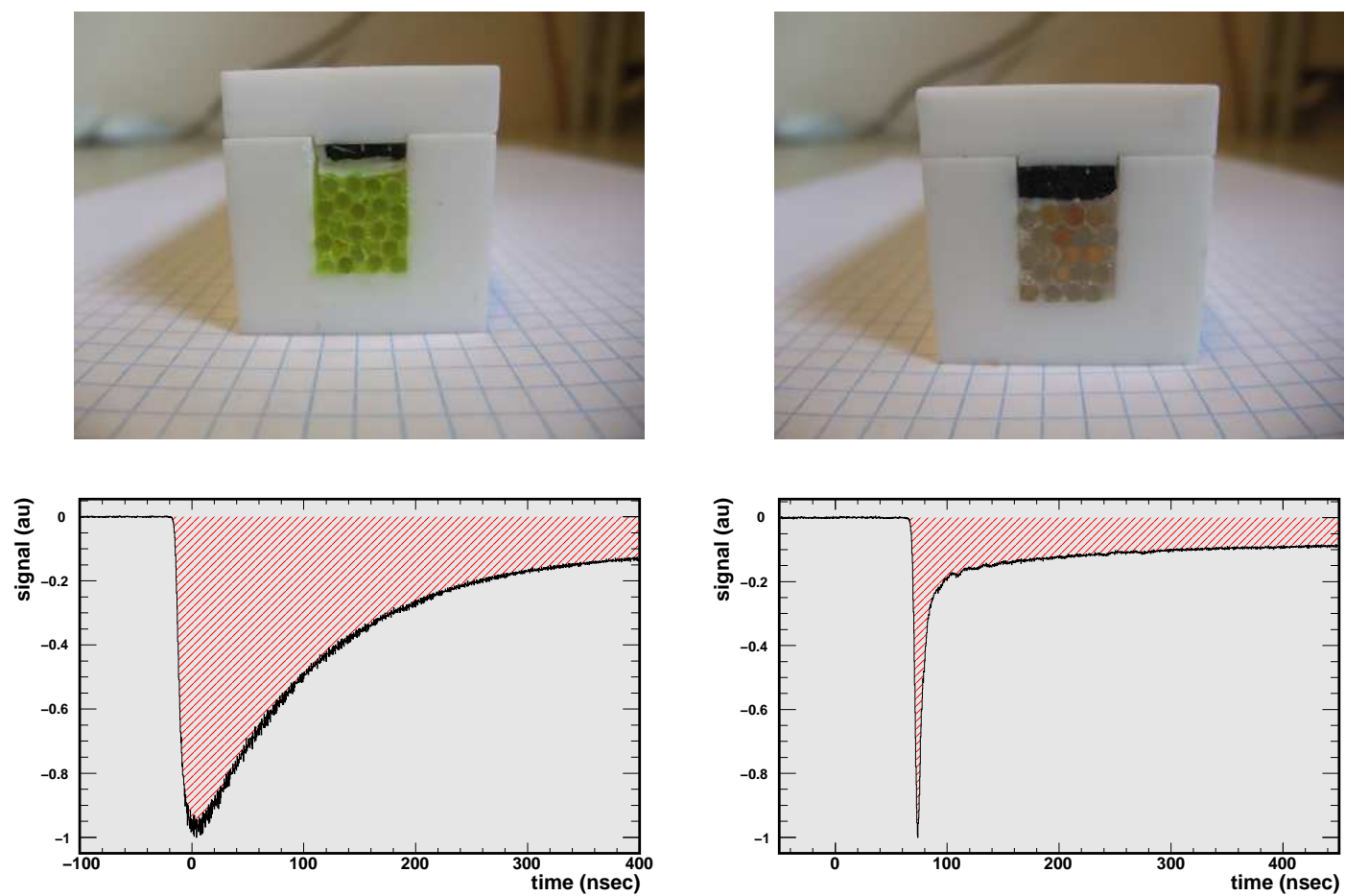

Figure 5. Bundles of Ce doped (top left) and undoped (top right) LuAG fibers and corresponding typical signal pulses recorded (bottom row). Each fiber measures $2 \mathrm{~mm}$ in diameter and $80 \mathrm{~mm}$ in length.

example of the series of measurements we perform on a sample to study its properties with respect to light transmission, excitation-emission, attenuation, diffusion etc for optical characterisation and further development of the crystal material [5].

\subsection{Testbeam activities}

Bundles of undoped and Ce doped LuAG crystal fibers have been exposed to electron beam at CERN SPS H4 beamline during short periods in 2009 and 2010. The fibers were read-out from both ends with photomultiplier tubes. The setup was temperature controlled and could be rotated so that the beam particles can traverse the fibers at given angle. A bundle of 20 fibers, with dimensions of $2 \mathrm{~mm}$ in diameter and $80 \mathrm{~mm}$ in length each, wrapped in a teflon box can be seen in figure 5. The corresponding signal pulses recorded are also shown. They are typical Cherenkov and scintillation light pulses as expected respectively. Some results of the data analysis are presented in figure 6 and 7. Figure 6 shows the left-right asymmetry of the recorded signal for different angles of orientation of the fibers with respect to the axis of the incident beam and for different setup configurations and samples. The undoped LuAG fibers show strong asymmetry as expected from a Cherenkov based detector due to the directionality of the signal generation mechanism. A maximum or minimum value of the asymmetry is expected at angles $33^{\circ}$ or $-33^{\circ}$ corresponding to the complement to $90^{\circ}$ of the Cherenkov emission angle in LuAG. A behavior of this type was observed for lead tungstate crystals as reported in [6] and [7]. The reason we do not observe a similar behavior with the bundle of LuAG fibers is the following: the fibers under study had short 


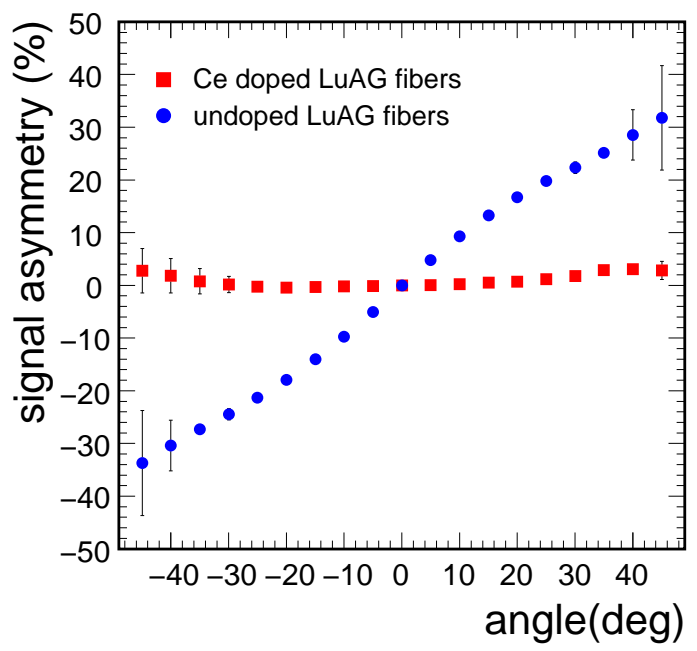

Figure 6. Normalised left-right asymmetry of recorded signal with bundles of Ce doped (square symbols) and undoped (round symbols) LuAG fibers for different orientation angle with respect to the incoming electron beam.
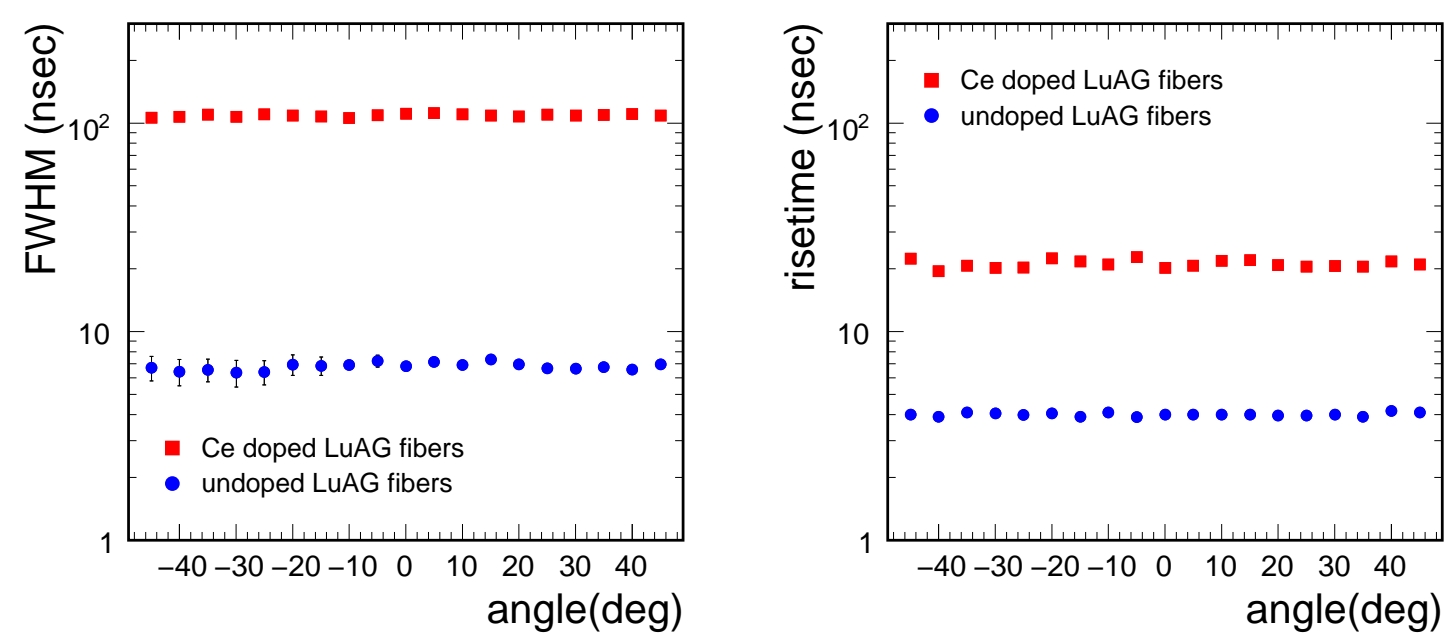

Figure 7. Full-width at half-maximum and risetime of average signal pulses recorded with bundles of $\mathrm{Ce}$ doped (solid symbols) and undoped (open symbols) LuAG fibers for different orientation angle with respect to the incoming electron beam.

length $(80 \mathrm{~mm})$ and when inclined with respect to the beam axis at angles larger than about $30^{\circ}$ the glass windows of the photomultiplier tubes were positioned inside the beam path. The particles that traverse through the windows generate also Cherenkov light that cannot be separated from the one that comes from the crystal fibers. Given the small overall fiducial volume of the bundle this signal is larger or comparable in size to the Cherenkov signal that comes from the fibers. This effect is causing the recorded asymmetry to appear monotonically rising and also is degrading the quality of 

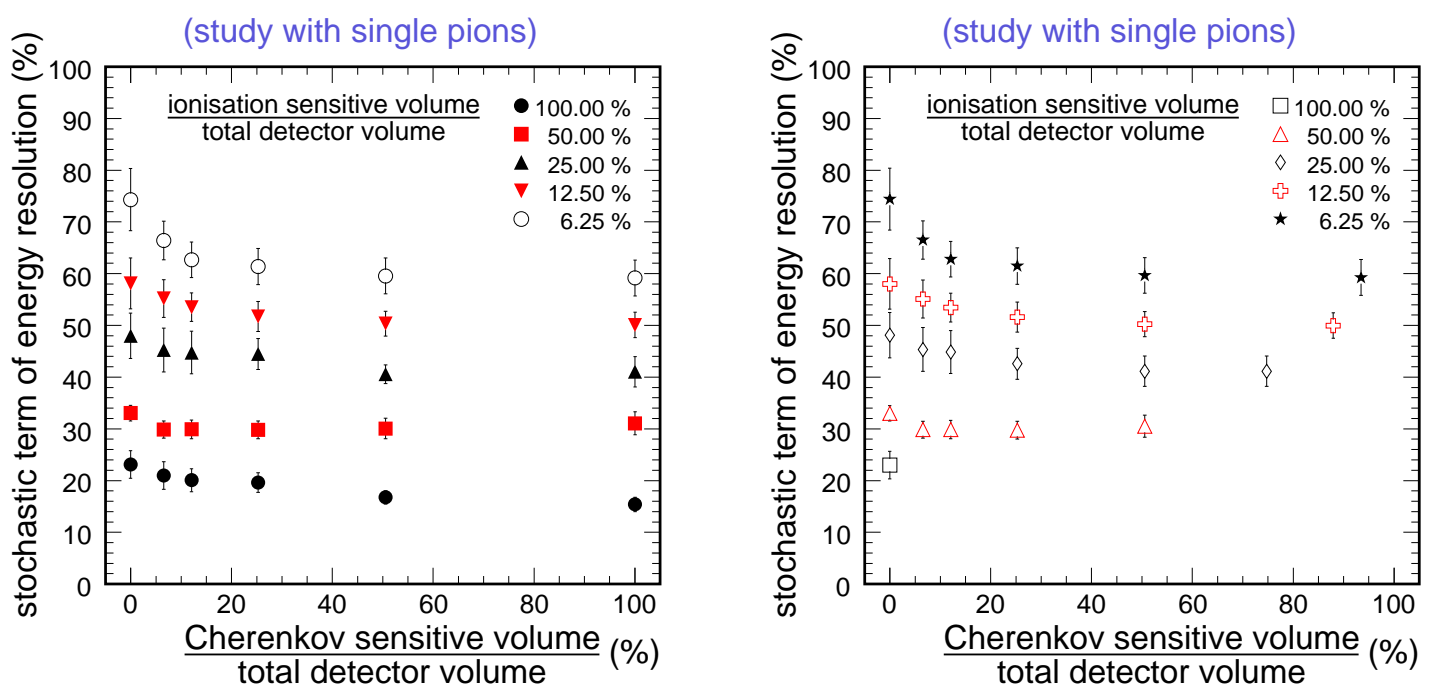

Figure 8. Simulated performance, in terms of the energy resolution's stochastic term, of $4.3 \times 4.3 \times 8.6 \lambda_{I}^{3}$ dual readout calorimeters with various sampling configurations of ionisation and Cherenkov signal readout. The sensitive volume is composed of fibers that can produce both scintillation and Cherenkov signals (left) or is composed of two types of fibers that produce either scintillation or Cherenkov signal respectively (right).

the data as can be seen by the significantly larger error bars for the points that correspond to larger angles of inclination. The effect though is present for both the doped and the undoped fibers it is much more pronounced in the case of the undoped fibers due to the much lower Cherenkov light yield compared to the scintillation light yield of the Ce doped fibers. The Ce doped fibers show less or no asymmetry as expected since the scintillation light is isotropically emitted. The full-width at half maximum of the average pulses, of the order of $100 \mathrm{nsec}$ for scintillating fibers and $7 \mathrm{nsec}$ for the Cherenkov ones, and the rise time, about $20 \mathrm{nsec}$ and $5 \mathrm{nsec}$ respectively, can be seen in figure 7. Further studies included the temperature dependence of the behavior of the fibers [7]. Similar studies are also done at a testbench using cosmic muons. So far we have performed small scale tests equivalent to the level of a single calorimetric channel, with respect to volume of sensitive material. Our plans in the near future include the construction and study of a multichannel module.

\subsection{Simulation studies}

We are performing systematic scannings of the parametric space with design parameters under study such as: transverse and longitudinal granularity, sampling frequency and readout fraction, total length of calorimeter, mixture of conventional and dual readout components, corresponding composition etc [10]. In the following we report on some of these simulation studies.

Figure 8 summarises the results from a set of case studies with a $4.3 \times 4.3 \times 8.6 \lambda_{I}^{3}(\approx 1 \times$ $\left.1 \times 2 \mathrm{~m}^{3} \mathrm{LuAG}\right)$ calorimetric volume and for different readout fractions of the scintillation and Cherenkov signals as denoted by the ratio of the ionisation sensitive volume, or the Cherenkov sensitive volume respectively, to the total detector volume. The studies are with single pions in the energy range from 5 to $50 \mathrm{GeV}$. The simulation code is based on Geant4 toolkit [8]. A readout unit 

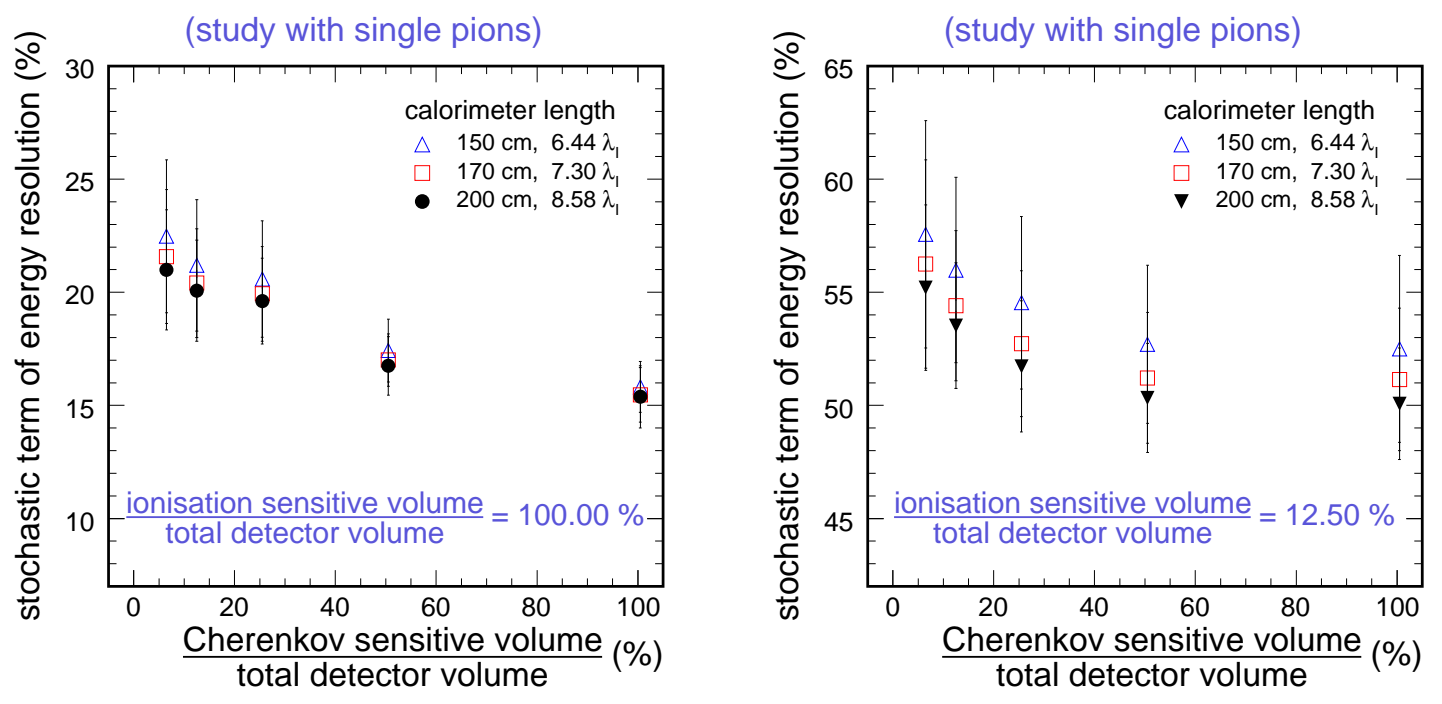

Figure 9. Simulation results for various calorimeter lengths and with ionisation readout fraction of $100 \%$ (left) and $12.5 \%$ (right).

is composed of a $4 \times 4$ matrix of $5 \mathrm{~mm}$ in diameter crystal fibers. Various sampling configurations of scintillation and Cherenkov signal readout are studied, covering the range of readout fractions $\left(=\frac{\text { sensitive volume }}{\text { total detector volume }}\right)$ from $6.25 \%$, where 1 out of 16 fibers is readout, up to $100 \%$, where all fibers are readout. The readout fractions of the scintillation and Cherenkov signal can be independently and arbitrarily set when assuming that the fibers can produce both signals (cases shown in the lefthand side plot of figure 8). To realise such a detector efficient methods to separate scintillation and Cherenkov light coming from the same crystal have to be developed. A sufficient degree of separation can be achieved with techniques based on the time and spectral properties of the Cherenkov signal. First application of such techniques for dual readout calorimetry and further discussion on the subject can be found in [6] and [9]. The figure shows the performance in terms of the stochastic term of the energy resolution after the dual readout technique, as described in [2], is applied. The left most series of points, i.e. $\frac{\text { Cherenkov sensitive volume }}{\text { total detector volume }}=0$, represent the resolution performance of a conventional detector i.e. without any readout of the Cherenkov signal and so where the dual readout correction scheme is not applied. We observe an improvement in the energy resolution achieved with the dual readout technique compared to conventional single signal readout and with a trend of improvement being relatively more significant as we approach cases with larger sampling readout fractions. The results obtained with fibers that produce both signals (figure 8 left-hand side plot) are almost identical to the ones that correspond to simulation of two types of fibers with separate functionalities (figure 8 right-hand side plot). This is expected and is due to the very high sampling frequency (fibers of $5 \mathrm{~mm}$ in diameter). For the case of a fully homogeneous calorimeter our current studies predict a stochastic term of the energy resolution as good as $23 \%$ with single signal readout and around $15 \%$ for dual readout. The influence of the total length of the calorimeter is shown in figure 9 for two cases of scintillation sampling readout of $100 \%$, i.e. the case of an homogeneous calorimeter, and $12.5 \%$ respectively. Figure 10 shows the results from a similar study but with the front part of the calorimeter being homogeneous in all cases up to a 

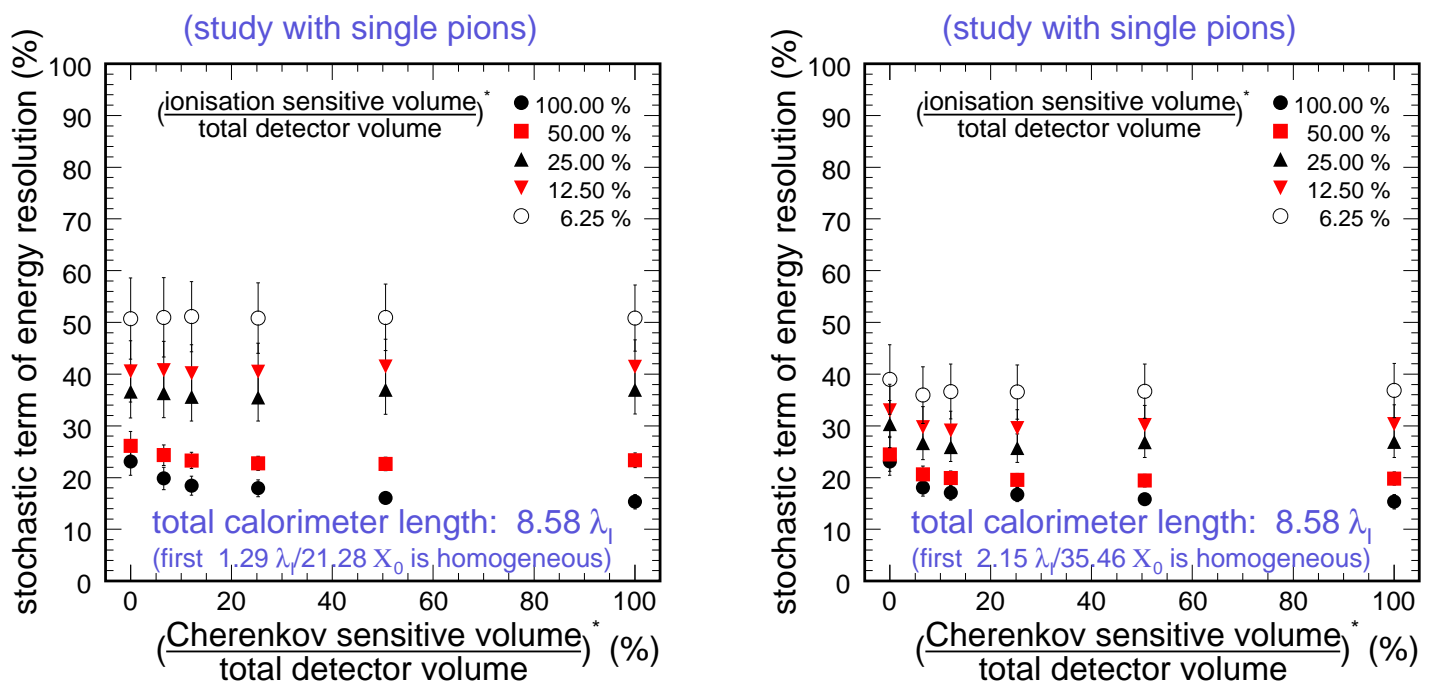

Figure 10. Simulated performance, in terms of the energy resolution's stochastic term, of $4.3 \times 4.3 \times 8.6 \lambda_{I}^{3}$ dual readout calorimeters with various sampling configurations of ionisation and Cherenkov signal readout and with the front part of the calorimeter being homogeneous in all cases up to a depth of $1.3 \lambda_{I}$ (left) and $2.1 \lambda_{I}$ (right), respectively. The ratios $\left(\frac{\text { ionisation sensitive volume }}{\text { total detector volume }}\right) *$ and $\left(\frac{\text { Cherenkov sensitive volume }}{\text { total detector volume }}\right) *$ refer to the part of the calorimeter that is not homogeneous.

depth of $1.3 \lambda_{I}\left(=21.3 X_{0}\right)$ and $2.1 \lambda_{I}\left(=35.5 X_{0}\right)$, respectively. We observe that the performance, in terms of the energy resolution's stochastic term, in all cases improves significantly and with the improvement being larger when larger fraction of the calorimeter volume is homogeneous. Further simulation studies to investigate other parameters, besides the stochastic term, that represent the various aspects of a calorimeter, such as the $\frac{e}{h}$ ratio, are planned.

Similar systematic studies combined with very preliminary cost considerations and technical challenges to be addressed help us to evaluate the performance trends, to understand any showstoppers and to proceed from an ideal case to a realistic detector. Figure 11 shows a preliminary effort to show such performance vs cost trends provided the oversimplified assumption that the total cost is proportional to the total sensitive volume. Two schemes are considered, single and dual readout, with or without homogeneous front part. In general if the performance goal for the next generation of calorimetric systems is set to a value equal or better than $30 \%$, with respect to the stochastic term of the energy resolution, then this goal can be achieved in various cases. A dual readout scheme with $30 \%$ or more sensitive volume can achieve it and so does a fully homogeneous detector with single readout. Given this large performance potential further rigorous feasibility studies and R\&D on these areas are well justified to be pursued. Within this context more detailed simulation studies are under way in order to investigate effects such as the fiber to fiber inhomogeneity, the light collection efficiency per readout channel etc. and how these could affect the expected performance.

\section{Outlook}

We discussed the meta-crystals concept an approach that consists of using undoped and Ce doped LuAG crystal fibers as the active medium to build a dual readout calorimeter. We presented some 


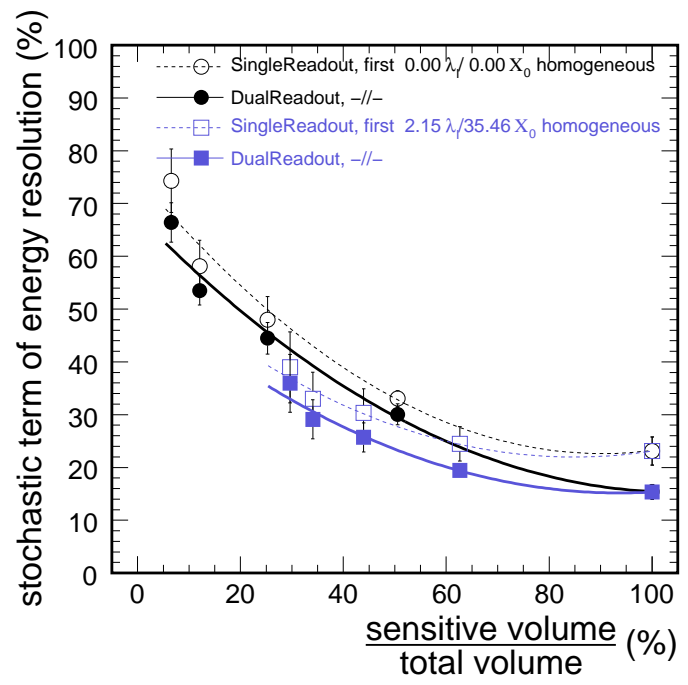

Figure 11. Simulated performance, in terms of the energy resolution's stochastic term, of $4.3 \times 4.3 \times 8.6 \lambda_{I}^{3}$ single or dual readout calorimeters with various sampling configurations of ionisation and Cherenkov signal readout.

aspects of the comprehensive $\mathrm{R} \& \mathrm{D}$ effort that has been established in our group with respect to material development and characterisation, fiber crystal production, first small scale testbeam activities and simulation studies for systematic scanning of the design parameters of a calorimeter. The simulation results for various generic schemes show very promising performance expectations. More detailed studies for a realistic detector design, to be experimentally studied, are planned.

We are at an early stage of development of such a concept with many design issues that should be studied further and which need rigorous R\&D and prototyping. Open questions and challenges include among others the optimisation of crystal fiber production in large quantities and related cost drivers, development of a readout scheme with diffractive optics or micro lenses, construction challenges and scale-up problems. These can only be answered through the usual phase of prototype development, test and study of 1 permille to 1 percent and 10 percent modules of the final detector. Our initial studies on some of these open issues are to be reported in the near future.

\section{Acknowledgments}

We would like to thank A. Para and H. Wenzel for valuable discussions.

\section{References}

[1] CALICE collaboration, J. Repond et al., Design and Electronics Commissioning of the Physics Prototype of a Si-W Electromagnetic Calorimeter for the International Linear Collider, 2008 JINST 3 P08001 [arXiv: 0805.4833].

T.C. collaboration, C. Adloff, Y. Karyotakis, J. Repond, A. Brandt et al., Construction and Commissioning of the CALICE Analog Hadron Calorimeter Prototype, 2010 JINST 5 P05004 [arXiv: 1003.2662]. 
[2] N. Akchurin, K. Carrell, J. Hauptman, H. Kim, H. Paar et al., Hadron and jet detection with a dual-readout calorimeter, Nucl. Instrum. Meth. A 537 (2005) 537.

[3] P. Lecoq, New crystal technologies for novel calorimeter concepts, J. Phys. Conf. Ser. 160 (2009) 012016 in Proceedings of XIII International Conference on Calorimetry in High Energy Physics (CALOR2008), Pavia, Italy, 26-30 May 2008.

[4] B. Hautefeuille et al., Shaped crystal growth of $\mathrm{Ce}^{3+}$-doped $\mathrm{Lu}_{2(1-x)} \mathrm{Y}_{2 x} \mathrm{SiO}_{5}$ oxyorthosilicate for scintillator applications by pulling-down technique, J. Cryst. Growth 289 (2006) 172.

[5] E. Auffray et al., LuAG material for dual readout calorimetry at future high energy physics accelerators, in Proceedings of the IEEE Nuclear Science Symposium and Medical Imaging Conference (2009 NSS/MIC), p.2245.

[6] N. Akchurin, Contributions of Cherenkov Light to the Signals from Lead Tungstate Crystals, Nucl. Instrum. Meth. A 582 (2007) 474 [arXiv:0707.4013].

[7] E. Auffray et al., Dual readout with PWO crystals and LuAG crystal scintillating fibers, IEEE Trans. Nucl. Sci. 57 (2010) 1454, in Proceedings of IEEE-10th International Conference on Inorganic Scintillators and their Applications (SCINT 2009), Jeju Island, Korea, June 8-12 2009.

[8] GEANT4 collaboration, S. Agostinelli et al., GEANT4: A Simulation toolkit, Nucl. Instrum. Meth. A 506 (2003) 250.

[9] N. Akchurin, L. Berntzon, A. Cardini, R. Ferrari, G. Gaudio, et al., Dual-Readout Calorimetry with Lead Tungstate Crystals, Nucl. Instrum. Meth. A 584 (2008) 273 [arXiv: 0707.4021$].$

[10] G. Mavromanolakis, E. Auffray and P. Lecoq, Calorimetry with meta-crystals, J. Phys. Conf. Ser. 293 (2011) 012082. 